\title{
ON THE SO-CALLED
}

\section{PARTIAL DISLOCATION OF THE HUMERUS.}

\author{
BY \\ EDMUND OWEN, F.R.C.S., \\ AGSISTANT SURGEON TO, AND DEMONSTRATOR OF ANATOMY AT, \\ ST. MARY'S HOSPITAL. \\ COMONONIOATED BY \\ GEORGE GREEN GASCOYEN, F.R.C.S.

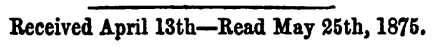

Evre at the present day opinion is divided as to whether a partial dislocation of the humerus should be included in the classical description of dislocation of that bone or not For my own part, I cannot understand the nature of an injury which would be sufficient to force the head of the bone through the upper part of the capsular ligament without causing fracture of one or both of the overlying scapular processes.

Examples of dislocations of the humerus with fracture of the coracoid processes have been recorded in the 'Transactions' of this Society by Mr. Holmes and Mr. Prescott Hewett. Such injuries, however, would not be included in the generally accepted definition of the "partial dislocation."

But even supposing that an injury had forced the head of the humerus into immediate contact with the under surface 
of the acromion process, why, we may ask, should it remain there? It cannot be from the bone being grasped below the equator of the head by the ruptured capsular ligament, for there is not sufficient room between the capsule and the process for the head to have passed far enough out of the cap. sule for such a condition to have resulted.

In the two well-known cases to which I am about to refer, and upon which the usual description of the partial dislocation has been founded, the capsule and the surrounding muscles were described as being entire, or else the ligament was "slightly ruptured." There has never been, so far as I can ascertain, any unequivocal instance of a subluxation of the humerus, and those authors who describe such a lesion base their account of it either upon the description given by Sir Astley Cooper in his grand work upon fractures and dislocations of the joints, or else upon the account of a peculiar condition of a shoulder-joint given by $\mathrm{Mr}$. Soden in the 'Transactions' of this Society for the year 1841. It is my endeavour in this paper to show that the changes noticed in these preparations were the result of disease and not of accident. And in support of this I shall describe the right and left shoulder-joints of two very old female subjects which were examined by me in the dissecting room of St. Mary's Hospital during the last two winter sessions. The right joints bore a very close resemblance to that described by Sir Astley Cooper, whilst both the left joints corresponded very closely with that which came under the notice of $\mathrm{Mr}$. Soden. It must be admitted that it is hardly likely that both these old women could have been subjects of partial dislocations of both humeri, the result of accidental violence.

Before going further, however, I would like to point out briefly the views held by various authors on the subject of the partial dislocation. Amongst those who admit it are-

Sir William Fergusson, who instances Soden's case, and who also describes specimens which he had examined in the dissecting room; of the latter, however, Sir William says, "Whether these effects were the result of violence or of gradual change, I cannot say." 
Erichsen, who gives Soden's case, and who believes that the subluxation can only take place when the tendon of the biceps is displaced from its groove or ruptured.

James Lane, in Cooper's 'Dictionary of Practical Surgery,' who quotes Soden's case, and one which was described by Mr. Alfred Smee, and to which I shall refer further on ; and

Pirrie, who quotes Sir Astley Cooper's case, and another dissecting-room specimen.

On the other hand, Hamilton believes that the anatomical structure of the joint renders the occurrence of such an injury exceedingly improbable, if not impossible, whilst

Flower and Hulke, in Holmes's ' System of Surgery,' deny that the partial dislocation as the result of accident has ever been satisfactorily proved, either in the living or dead subject.

Robert Adams, in 'Todd's Cyclopædia,' attributes the lesion described as subluxation by various authors, to chronic disease or congenital malformation.

Then, having given an account of my own preparations, I will place their analogues in close connection.

Case 1.-On cutting across the right deltoid of the first subject an enormous bursa was discovered in communication with the articular synovial membrane through a well-defined elliptical opening in the upper part of the capsule. Through this aperture the head of the humerus was enabled to articulate with the under surface of the acromion process, where a smooth articular facet had become developed. The muscles surrounding the joint were entire. The capsule was attached to the coracoid and acromion processes, and there was a considerable development of cartilage and of vascular fringes in different parts of the joint. The circumference of the head of the humerus was increased in size by the growth of tuberculated piec es of bone and cartilage'; and as the articular cartilage was thickened in some places, and eroded in others, the globular mass of bone was of a very irregular shape. The intra-articular portion of the biceps was ruptured, and the lower part of the tendon was displaced from the bicipital groove and adhered firmly to the lesser tuberosity. 
CASE 2.-The right joint of the second subject was very similar to that just described. The subdeltoid bursa was very large, and the tendon of the biceps was ruptured, and was adherent to the upper part of the bicipital groove. The head of the humerus was much enlarged by osseo-cartilaginous deposit, and was pressing up underneath the acromion process and the coraco-acromial ligament, and there was a corresponding erosion of the articular cartilage. The two surfaces of bone, however, were not in immediate contact, as the humerus had not sufficiently worked its way through the loose capsular ligament when death had occurred.

Here follows the account of Sir Astley Cooper's specimen, which was obtained from the dissecting room of St. Thomas's Hospital in the year 1819. The account is transcribed from Sir Astley's work almost literally :- "The head of the humerus was placed more forward than is natural. The tendons of those muscles which are connected with the joint were not torn, and the capsular ligament was found attached to the coracoid process of the scapula. The head of the os humeri was situated under the coracoid process which formed the upper part of the new glenoid cavity. The natural rounded form of the head of the bone was much altered, it having become irregularly oviform with its long axis from above downwards. There was considerable deposit of cartilage in the rest of the glenoid cavity and upon the head of the humerus. The long head of the biceps muscle seemed to have been ruptured near to its origin (at the upper part of the glenoid cavity), for at this part the tendon was very small, and had the appearance of being a new formation." (The rest of the short description is of no material inportance; the right joint does not seem to have been examined.)

These three descriptions agree in their most important features : viz., in the alteration in shape and position of the head of the humerus (the enlarged head lying against one of the scapular processes), in the rupture of the biceps tendon, in the new growths in the joint, and in the uninjured condition of the small rotator muscles. 
The left joints of my two subjects presented many features corresponding to those of the right side, but the disease was in all respects less advanced, so that the tendon of the biceps, though worn very fibrous and ragged, had not yet given way. In subject No. 2 it was very thin and frayed out. In both cases, however, it had been displaced from the bicipital groove by a new osseous growth, and was dislocated on to the lesser tuberosity of the humerus, where it played in a separate sheath of fibrous tissue and synovial membrane. These specimens closely resemble that described by Mr. Soden, of which a short account is here subjoined.

In May, 1839, a rheumatic man, thirty-nine years of age, fell on to his right elbow whilst rising from nailing down a carpet. Great pain then came on in the corresponding shoulder, but he could raise his arm over his head, and went on working till the pain compelled him to desist. Next morning the joint was greatly swollen, but Mr. Soden satisfied himself that there was neither fracture nor dislocation. Three weeks afterwards, when the swelling had disappeared, Mr. Soden gave the joint another examination, when he experienced a crepitating sensation upon moving the limb. He also found that the arm could be but little raised, on account of the great tuberosity coming into contact with the acromion. There was also undue prominence in front.

Six months afterwards the man died in the Bath Hospital of fracture of the base of the skull, and a subsequent examination of the joint gave the following results :-The capsule, but slightly ruptured, was thickened and adherent. The synovial membrane was vascular and inflamed. The head of the humerus was in contact with the acromion process, and its articular cartilage was ulcerated at that spot. The tendon of the long head of the biceps was entire, and, dislocated from the bicipital groove, lay in a synovial sheath upon the lesser tuberosity of the humerus. The preparation is now in the museum of King's College, where I have examined it, as far as $I$ am able. The head of the bone, as in the other cases, is considerably enlarged, and its circumference rendered irregular by new bony or cartilaginous 
growths. In the adjoining bottle in the museum (1341 (4)) is a preparation from Sir William Fergusson's collection. 'It is "described in the catalogue as "an old dislocation ;" and as the articular vessels are filled with injection, $I$ infer that it is one of those dissecting-room specimens of whose nature the great surgeon gave so cautious an opinion. It seems to be in every respect similar to Sir Astley Cooper's preparation, and to the two right joints which $I$ have just described. It is not a little strange that they should, all four, be dissectingroom specimens.

Soden's preparation, then, and my two left joints, agree in almost every respect. They differ from Cooper's and from my right joints only in this, that the disease is less advanced. It is not unlikely that the right joint is more prone to disease than the left. In a little time the tendon would, in my cases at least, have worn through. As it was, it was merely dislocated, as described by Soden, and the cause of this dislocation I attribute to a gradual filling up of the bicipital groove.

A most important point in connection with my second pair of shoulder-joints is, that the knee-joints of the same subject were also affected with a chronic arthritis. The patella shows considerable bony deposit, whilst its posterior surface and the trochlear aspect of the femur are in places entirely denuded of cartilage, and are worn by friction in longitudinal streaks. [These parts of the left side were exhibited.]

Mr. Callaway gives the account of twenty-two (22) preparations of the shoulder.joint in which there has been found either a dislocation or a rupture of the tendon of origin of the biceps, and states his belief that the partial dislocation of the humerus is due to one of these peculiar conditions.

He includes in this series the preparations of Sir Astley Cooper and of Mr. Soden, and also some which Robert Knox had described in the 'London Medical Mirror,' which were in every way similar to those now before the Society. He thought that such lesions were rare, or that they were rarely 
reported. They were, I apprehend, all dissecting-room specimens.

Shortly after, and in the same publication ("London Medical Mirror,' 1828), Mr. Stanley gave an account of three similar specimens. Callaway includes these also, and a dissecting-room preparation which was described in the 'Lancet' for 1845 by Mr. Alfred Smee, of the Aldersgate School of Medicine. This last joint is of further interest, for the disease in it was so far advanced that not only was the tendon of the biceps ruptured and adherent to the head of the humerus, but the greater tuberosity was almost all articular, and was of necessity deprived of its muscular connections. $\mathrm{Mr}$. Smee could hear of no case like his but that of $\mathrm{Mr}$. Soden; but this, he went on to say, differed from his own, in that the tendon of the biceps was merely dislocated. With the exception of Mr. Soden's case, however, he considered that his own was without precedent.

The great similarity existing between my own shoulderjoint preparations (which are clearly affected by a chronic disease, probably of rheumatic origin) and those to which I have referred, leads me to this conclusion, that the specimens described by Cooper and Soden were examples of the same pathological change, and cannot, therefore, be taken as instances of "partial dislocation" in the proper sense of the term. And I am of opinion that, until some unequivocal example of the partial dislocation be adduced, the description of the lesion as the result of injury should be omitted from our text-books, more especially since the anatomical structure of the joint contra-indicates the possibility of its occurrence. 